\title{
Considerations for the Mobile Web. Paradigm Shift
}

\author{
Romeo MARGEA, Camelia MARGEA \\ West University of Timisoara \\ romeo.margea@e-uvt.ro, camelia.margea@e-uvt.ro
}

The paradigm shift around the Mobile phenomenon - the explosion of mobile online users -, Google's response to that trend and the what such changes entail further for website developers are issues around which this paper is built. In the first part of this paper we made an analysis of the literature in order to clarify certain terminology and trends of Mobile Web. Further we illustrate the practical aspects of implementing these technological issues for a faculty institutional website (Faculty of Economics and Business Administration within the West University of Timisoara). In the last part, we analyzed statistical data collected during last five years of operation of that website, emphasizing compliance with the trends in the field worldwide.

Keywords: Mobile-Friendly, Mobile-First, Mobile Websites, Google Analytics.

\section{1} Introduction

In recent years, the number of mobile devices (smartphones, phablets, tablets) and of mobile device users has grown considerably, impacting on user behavior, especially with respect to Web browsing habits.

To support this mobile trend, Google has undertaken and still undertakes numerous efforts, by continuously updating its search algorithm in order to meet the users' needs more effectively. On the other hand, according to Google's latest algorithms, website owners constantly struggle to optimize content in order to score higher in search results [1]. On the whole, these optimization efforts benefit both the indexing process and mobile users.

One of the reasons why Google offers priority to mobile-optimized web pages and favors the "mobile first" approach is that nowadays most users use their mobile devices to search online [2]. Mobile online searches have been growing yearly, with 2015 registering more mobile-based than desktop-based online searches [3].

Google states that "more Google searches take place on mobile devices than on computers in 10 countries including the US and Japan", however, without giving further details [4]. This fact, which seems to confirm Google's interest in mobile Web, has stimulated greater efforts toward offering users better mobile experience [5].

As depicted in Figure 1 timeline, Google made public its interest in mobile browsing as early as 2010, and the first major step was taken in October 2014, when the company introduced the Mobile Usability report in Webmaster Tools [6].

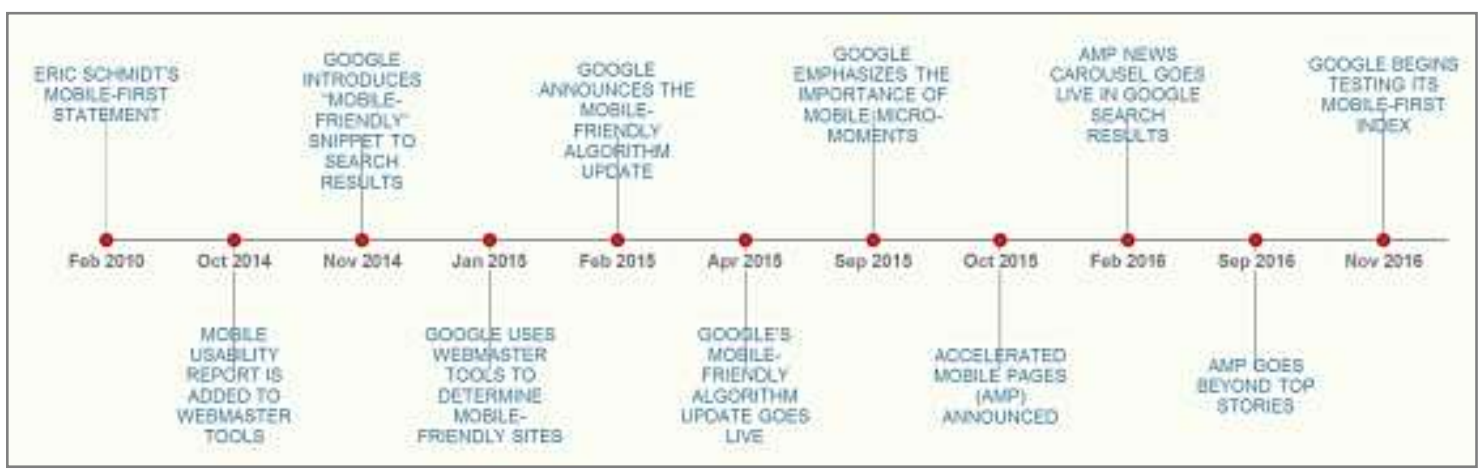

Fig. 1. Timeline of Google steps regarding mobile web

Of the many steps taken by Google in this direction [6], we would like to mention two which, in our opinion, have the strongest impact on mobile Web: 
- Mobile-friendly algorithm update Mobilegeddon (April 21, 2015);

- Mobile-first index (November 2016).

\section{Mobile-Friendly Algorithm Update}

On 21 April 2015, Google launched the Mobile-friendly algorithm update (MFAU), their new mobile ranking system (nicknamed Mobilegeddon), through which mobile search engine results pages (SERPs) are influenced by the website's "mobile friendliness" [7]. This update of the search algorithms only impacts mobile-based online searches, regardless of the language used or of the country of origin [8].

MFAU aims to significantly alter search results on mobile devices by giving priority to mobile-friendly instead of non-mobilefriendly websites. This change does not affect desktop/laptop-based searches. Google's intention is to facilitate access to mobilefriendly websites for mobile device users [1]. A mobile-friendly website is essentially a normal website which works well and is displayed properly on both desktop/laptop and mobile devices. The site can be shrunk down so as to be small enough to be displayed on a mobile device. Even if the user must zoom and scroll, the website is displayed and works properly. The experience may not be perfect, but the website can be decently displayed and browsed [9].

Due to MFAU, the websites whose owners failed to implement a strategy to adapt the sites themselves as well as their content to mobile device experience will rank lower than mobile-friendly websites [8].

In order to give website owners time to prepare for imminent changes and to give the world the chance to benefit from their advantages, Google made public its intention to introduce MFAU two months before it was due to be launched [10].

Following this statement, Google noticed a $4,7 \%$ growth in the number of mobile-friendly websites in March and April 2015. Fear of MFAU (hence the apocalyptic connotations of the label Mobilegeddon) and of potential traffic loss made many website owners upgrade them to mobile-friendly level [11].
A lot of people were persuaded that the April 2015 upgrade would have a devastating effect on businesses lacking mobile presence online [12], but this proved less serious than expected. Those monitoring ranking changes did not notice anything that would justify Google's statements according to which this update surpassed previous Panda or Penguin updates [13]. Although this update could have affected numerous queries, it did not impact online business as much as Panda and Penguin; in fact, the impact that could be measured proved negligible.

One of the causes of moderate impact could be the fact that the mobile-friendly algorithm is of the on or off type. This means that, since there are no measurable degrees of mobile friendliness in this algorithm, it does not matter how mobile-friendly the pages of a website are; what matters is simply whether they are mobile-friendly or not. At the same time, it is worth remembering that mobile friendliness is a feature noticeable at page level (page-bypage), not at website level [1]. This aspect is particularly important since many websites have subsections or specialized pages which are difficult to make mobile-friendly. Of course, it is important that all web pages be mobile-friendly, but should the conversion process be too complex, a decision can be made at page level (page-by-page), as the number of mobile users increases [14].

Although making changes in favor of mobile devices is important, Google still uses a wide range of signals to rank search results. Even though a web page of high-quality content is not compatible with mobile devices, it might still rank high if its content is of great value for a particular search.

As long as people keep using mobile devices to do Internet searches in higher and higher numbers, Google will give them priority (as we shall see in the next chapter - Mobile-first Index) while trying to give mobile users the best search experience.

\section{Mobile-First Index}

At the beginning of November 2016, Google took the second big step toward mobile experience by announcing and introducing Mobile- 
first index (MFI) to improve experience, whether on desktop or on mobile platforms [15].

MFI can be regarded as a direct response to the way people use Google nowadays, as Google noticed that not taking into account the mobile version of web pages in determining their quality can be problematic. Currently, most users use mobile devices for online searches whose results are often unsatisfactory due to scalability issues, annoying ads, etc., which appear when desktop versions of the websites in question are loaded onto mobile devices [5].

Before introducing the Mobile-first index, Google's search engine had given priority to the analysis of the desktop version of web page content over the mobile version in establishing the ranking on the result pages, although the search had originated from mobile devices [2].

MFI splits search results into separate versions for desktop and mobile, thus allowing to use the mobile version instead of the desktop version. The searches done on mobile devices no longer display desktop-based results and vice versa. The mobile index will become the main Google index, comprising mainly mobile web pages, which means that snippets, structured data as well as other types of content will appear among Google search results [5].

Two types of websites in particular are going to experience difficulties once MFI comes into effect [5]:

a) Desktop sites lacking mobile versions. Once MFI becomes the main index, Google will start to index websites (whether mobile or desktop versions) using mobile Googlebot. Although Google stated that they would continue to properly index desktop websites even if they lack a mobile version, these would not rank as well as before in the new index. On the other hand, Google stated that a functional desktop website can be better than a faulty or incomplete mobile version.

b) Different mobile and desktop website versions. Problems might arise when a mobile web page has less content than the corresponding desktop web page, which makes it impossible for algorithms to properly assess that web page. The solution is to make sure that both the mobile and the desktop version of the website have the same structure and content.

In this context, online businesses are constrained to adapt to this new algorithm and to give special attention to functionality in both desktop and mobile web pages. Even companies that operate in the field of mobile optimization and which anticipated this change have steps to take in order to prepare themselves for MFI. Any website that is not ready for these changes can experience problems and some of these websites might even need general revisions before MFI can become fully functional.

\section{What are the Available Technical Solu- tions to Be Mobile-Friendly?}

For website owners and developers, the paths toward mobile-friendliness and mobile-first index are many and sinuous. For them, the absence from the search results on mobile devices equals losing shares in business opportunities.

In order for a website to become mobilefriendly, one needs to take into account all the possible interactions between customers and a mobile device, which in turn should shape planning and structuring the website for mobile use. Browsing should be intuitive and the different elements of a website, such as buttons, menus and links should be accessible by touch instead of mouse [1].

Website owners and developers have three different configurations at their disposal in order to create mobile websites, all of which are approved by Google [16]:

- Responsive Web Design (RWD). This is a client-side type of approach, which uses the same HTML code on the same URL, irrespective of the users' browsing devices. However, the website is displayed differently, depending on the device screen resolution. This is the option approved and recommended by Google.

- Dynamic serving (aka Adaptive Deliv- 
ery). This is a server-side type of approach, which uses the same URL irrespective of the device, while dynamically generating different HTML code versions by detecting the browser's User Agent.

- Separate URLs (aka mDot sites). This is a server-side type of approach, which uses a different code for each device and a separate Web address for the mobile version (m.domain.com) - a subdomain or a path beginning with $\mathrm{m}, \mathrm{mob}^{*}$, iPhone, iPad or touch. This configuration detects the type of user device and then redirects to the corresponding web page by using HTTP redirects.

For those who do not yet have mobile-friendly websites, mDot can be a simple solution, but Google recommends that website owners use RWD. However, on their most important websites, Google implements RWD as well as Adaptive Delivery and mDot [8].

RWD, which still is the main trend when it comes to mobile-friendly websites, is about adapting a desktop website so that it fits smaller screens. RWD eliminates the need for an alternative mobile version of the site while allowing to host a single website which automatically adjusts itself to the resolution of the screen on which it is displayed [1]. In order for a website to be considered RWD compatible, it must meet the following criteria: it must use Media Queries, it must not allow horizontal scrolling and Viewport must be defined in a meta tag [8].

Although there are many differences between the various mobile website configurations, in order to establish whether a site is mobilefriendly, Google scans for and adjust the following elements [1] [17]:

- whether fonts scale or not in order to make reading easier on small screens, without the need for zooming;

- whether the elements that must be reached (i.e. buttons, links) are easy to use and spaced properly if surrounded by other elements;

- whether the website uses Adobe Flash or any other piece of software that is uncommon on mobile devices, and might not be displayed properly in mobile browsers;
- if there are separate mobile URLs, mobile users must be redirected from each desktop URL to the corresponding mobile URL;

- the ability to avoid irrelevant cross-links i.e. links to desktop-optimized web pages, displayed on the mobile version of the website and vice versa;

- if the website adapts to the screen resolution to display content, so that users do not have to scroll horizontally or zoom in to read the text displayed.

\section{The Configuration Chosen for the FEAA Website}

The website of the Faculty of Economics and Business Administration from the West University of Timisoara (http://feaa.uvt.ro) meets the mobile-friendly requirements (see at https://search.google.com/search-console/mobile-friendly?id=UEe-

XThhsQva8S6ujOptOQ the Google's Search Console Mobile-Friendly Test), as it is of the Responsive Web Design (RWD) type with several elements of Adaptive Delivery.

In order to make the first versions of the website mobile-friendly, we used the extension Mobile Joomla - a server-side type of solution, available at www.mobilejoomla.com. Subsequently, we adopted an RDW type of approach, which transfers the task of displaying the website properly on mobile devices to the template of the website.

The templates used are created by YOOtheme $\mathrm{GmbH}$ from Hamburg, Germany (http://yootheme.com), and are based on the $7^{\text {th }}$ generation of the Warp framework (made by the same company). Warp 7.x is a fast, responsive and lightweight cross-platform based on HTML5 and Bootstrap, with "Adaptive Delivery" features [18].

In what follows we will insist on the Adaptive features of the Warp framework used for the FEAA website. They allow to selectively display the various website elements or modules depending on the type of device used (desktop, tablet, smartphone), while functionalities are determined in the tab Modules, as shown in Figure 2. 


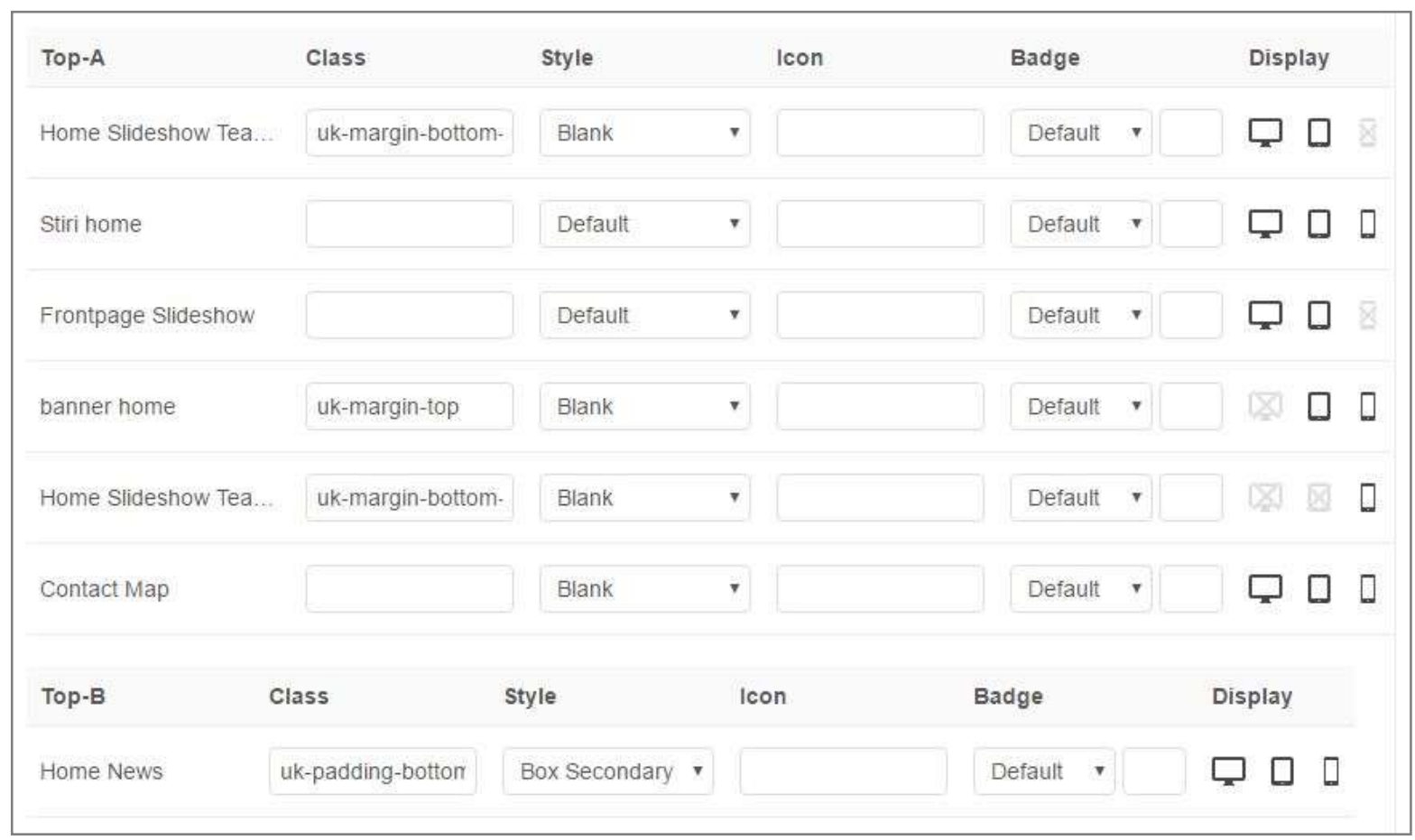

Fig. 2. Adaptive features of the Warp framework

For example, a desktop and a tablet can both display a large banner, while a smartphone can display a smaller banner. Thus, only the elements that need to be displayed on the device in question are sent to the user's browser and, in turn, this reduces the amount of data and, implicitly, the loading time on mobile devices.
Apart from the framework's Adaptive features, the FEAA website also uses a series of parameters of the Advanced Module Manager extension (www.regularlabs.com/extensions/advancedmodulemanager). This "must have" extension gives the system the possibility to selectively display any module, depending on the device, operating system and browser, etc.

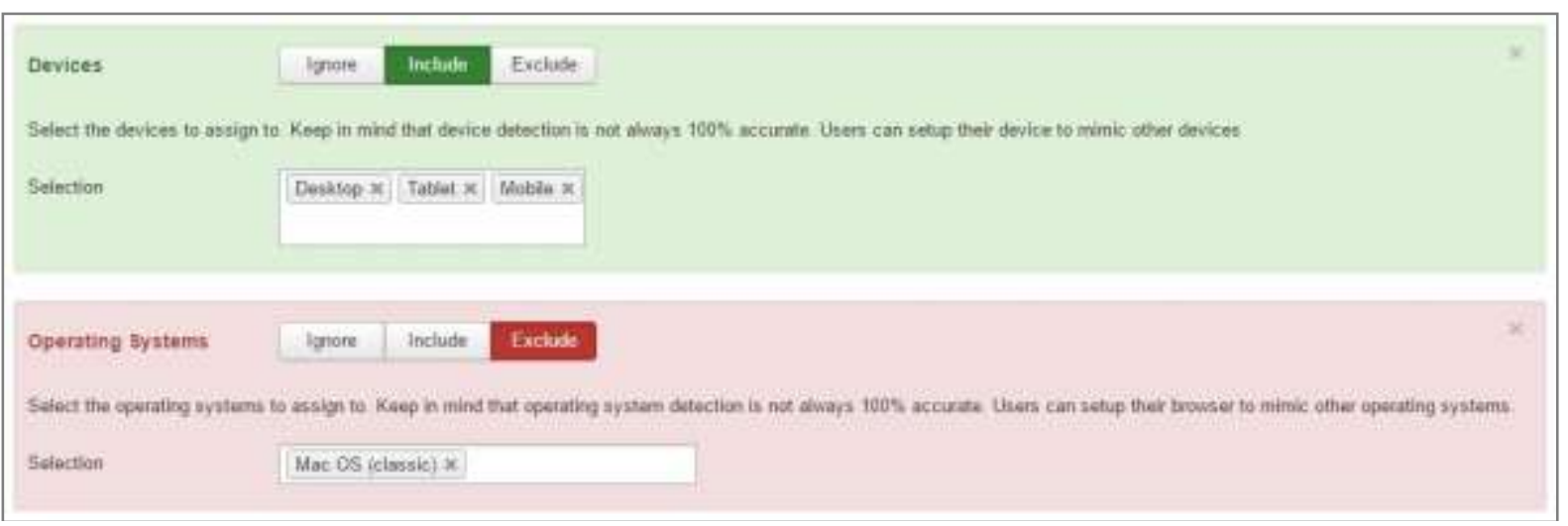

Fig. 3. Advanced Module Manager extension

\section{Mobile Internet Statistics}

Google's actions regarding mobile devices were not devoid of impact on the online environment and this is easily noticeable when analyzing several statistics for the two important moments presented in the first half of this paper: the Mobile-friendly algorithm update and Mobile-first index. 
6.1 The Moment of Mobile-Friendly Algorithm Update

We will begin by presenting the results of a study carried out by Saeteraas in April 2015, which analyzed the most popular websites at global level (Alexa Top 10000 Sites www.alexa.com), in order to see how they tried to respond to the diversity of devices and of browsers [8].

Saeteraas noticed that $86 \%$ of the top 100 websites were mobile-friendly. However, out of a total of 10,000 websites, only $72 \%$ tried to take mobile-friendly types of approaches. This means that the $28 \%$ remaining seemed to have done nothing in particular to become mobile-friendly although the algorithm had been announced two months before.

A surprising finding was that, despite Google's promoting RWD as the most mobile-friendly approach, most of the top websites opted for server-side approaches. In fact, $63 \%$ out of the first 10,000 websites used a server-side type of approach (mDot or Adaptive Delivery), and that percentage goes as high as $88 \%$ for the top 100 websites analyzed. An in-depth analysis of the mobilefriendly websites out of the total of 10,000 revealed that mDot was the most popular approach (40\%), thus surpassing even RWD (38\%) and Adaptive Delivery (22\%).

Another study similar to Saeteraas' was undertaken in June 2015 by Borodescu. It also took into account the Alexa Top 10,000 Sites from 5 different categories: News, e-Commerce, Tech, Business and Sport [19].

According to the results, almost $28 \%$ of the sites studied are responsive, while $26 \%$ opted for a second mobile URL. The difference between the two approaches was not as big as one might have expected. What is surprising, however, is that $40 \%$ of the websites analyzed had no mobile Web strategy whatsoever.
Another interesting aspect is that approximately $4 \%$ of the websites showed that Adaptive Delivery had been deemed the right strategy for mobile Web. 2\% of the websites analyzed chose to target their users through $i O S$ apps. Their number could, in fact, be higher, but the inconsistency in promoting apps makes them hard to track.

In March 2016, Borodescu carried out a new study on 10,000 Alexa sites, by keeping the same five key categories [12].

Out of the websites that, a year before, were not mobile-friendly, $25 \%$ showed signs that various mobile optimization strategies had been implemented. Most of these $(85 \%)$ had used RWD, $11 \%$ had chosen Adaptive and 4\% had opted for the mDot mobile version at a secondary URL.

As expected, the e-commerce website owners were the first to adapt, so that $31 \%$ of the ecommerce websites lacking mobile capabilities implemented some. Thus, in March 2016, $71 \%$ of the e-commerce websites had mobile presence, as opposed to 58\% in April 2015.

\subsection{The Moment of Mobile-First Index}

An important event related to the use of mobile devices for browsing that took place in October 2016 might have triggered the introduction of MFI by Google in November 2016. In this context, should not come as a surprise the data published in November 2016 by StatCounter - an independent Web analytics company whose Global Stats data relies on over 15 billion web page views per month, gathered from over 2,5 million websites, every 4 hours updated and made available [20]. According to that data, in October 2016, for the first time at global level, web page access on mobile devices surpassed those originating from desktops and laptops. 


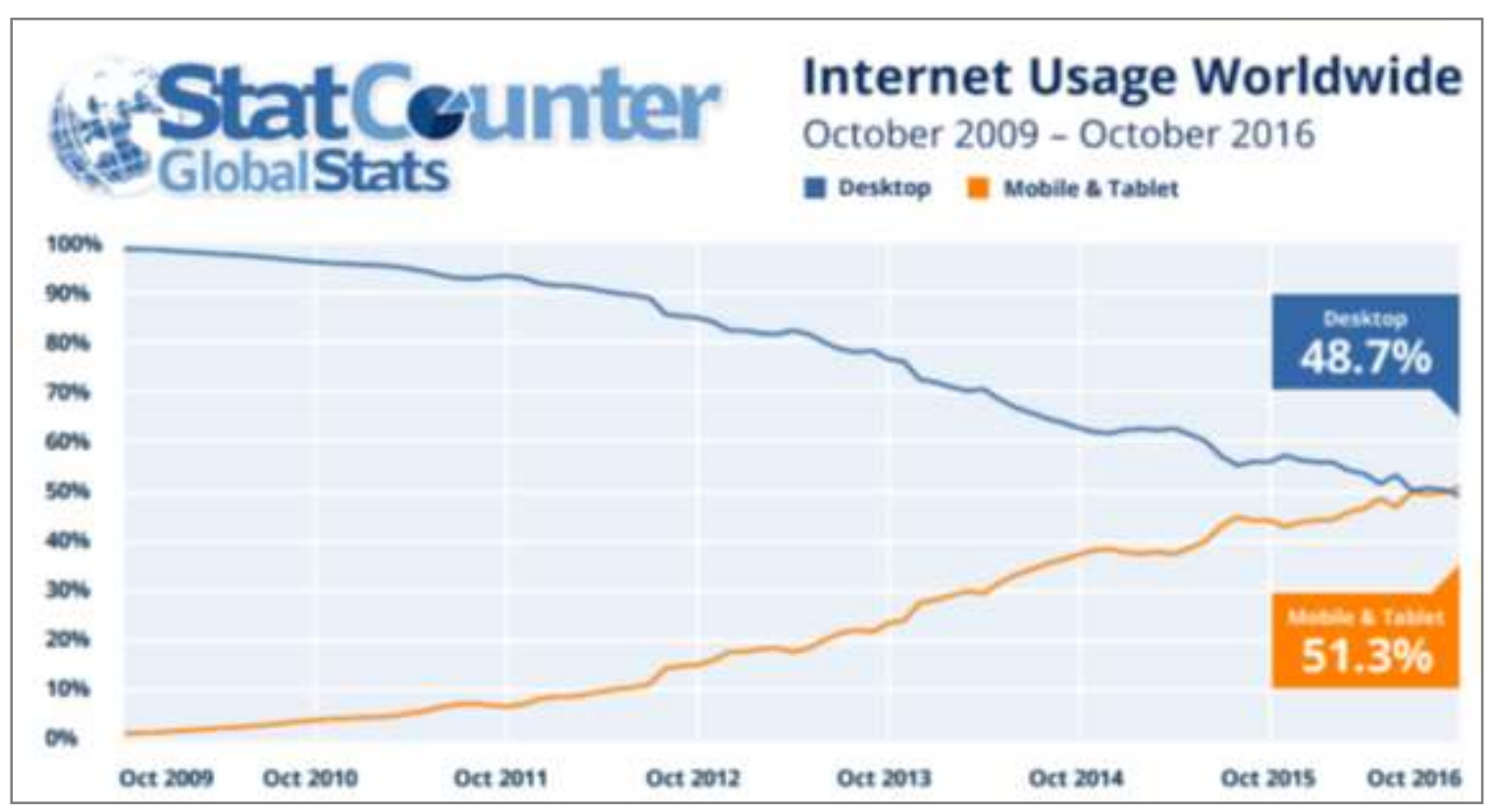

Fig. 4. Desktop vs. mobile Internet Usage worldwide trend October 2009-October 2016. Generated by statcounter.com

StatCounter noticed that, in October, $51,3 \%$ of the monitored web pages had been loaded on mobile devices as opposed to $48,7 \%$ on desktops and laptops. In 2010, these two represented less than 5\% and, in 2013, less than $25 \%$. From the mobile devices used, smartphones had accounted for $46,5 \%$ of the traffic, while tablets were down to $4,7 \%$ [21]. In spite of the rapid growth in mobile devices, desktops remain the main means for Internet use on mature markets such as the USA and Great Britain, but the gap is shrinking dramatically. Mobile Internet is much more popular in countries such as India, with a share of
$75 \%$.

In Great Britain, Internet access on desktop platforms accounts for $55,6 \%$, while mobile access for $44,4 \%$. In the USA, desktops still maintain a $58 \%$ share in Internet use as opposed to $42 \%$ for mobile devices [20].

The published figures lead to the conclusion that market maturity determines the dominant means of accessing the Internet. Mobile platforms are by far the first choice when it comes to emergent markets. More mature markets still favor desktops, but the gap keeps shrinking.

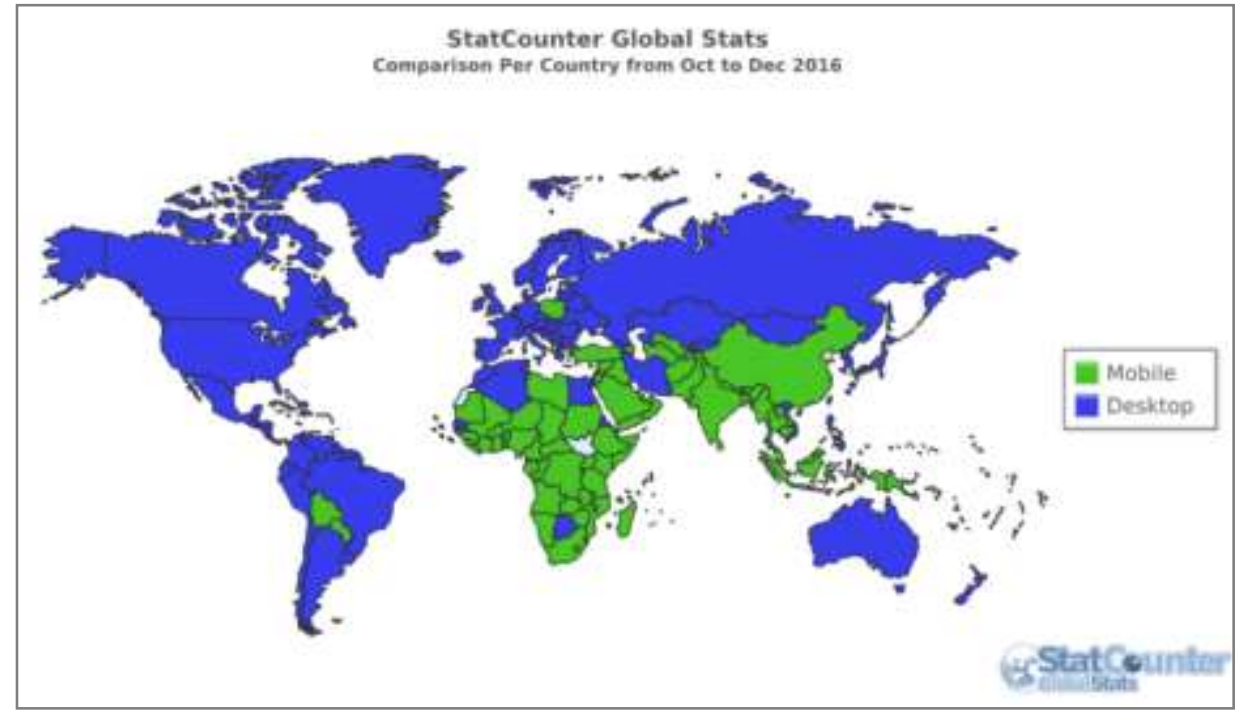

Fig. 5. Desktop vs. mobile Internet Usage worldwide in October-December 2016. Generated by statcounter.com 
With regard to the aspects presented above, Aodhan Cullen, CEO, StatCounter, made several statements: "This should be a wake-up call especially for small businesses, sole traders and professionals to make sure that their websites are mobile friendly. Many older websites are not." "Mobile compatibility is increasingly important not just because of growing traffic but because Google favors mobile friendly websites for its mobile search results." [20]

It is worth remembering that the data presented exclude the use of mobile apps such as Facebook and WhatsApp, which account for a significant percentage of mobile Internet use.
How our country and Europe fits into the worldwide mobile landscape? The Figure 6 shows the percentage of instances of Internet access on desktop vs. mobile device in Romania between October 2011 and December 2016. Further, to be able to take the global situation into account, we began by an in-depth analysis of the period October-December 2016, which allowed us to notice that the number of desktop/laptop Internet access instances was still higher than for mobile devices, at $67,39 \%$. The whole situation is similar to Italy's $(67,63 \%$ desktop $)$ and Germany's (69,08\% desktop).

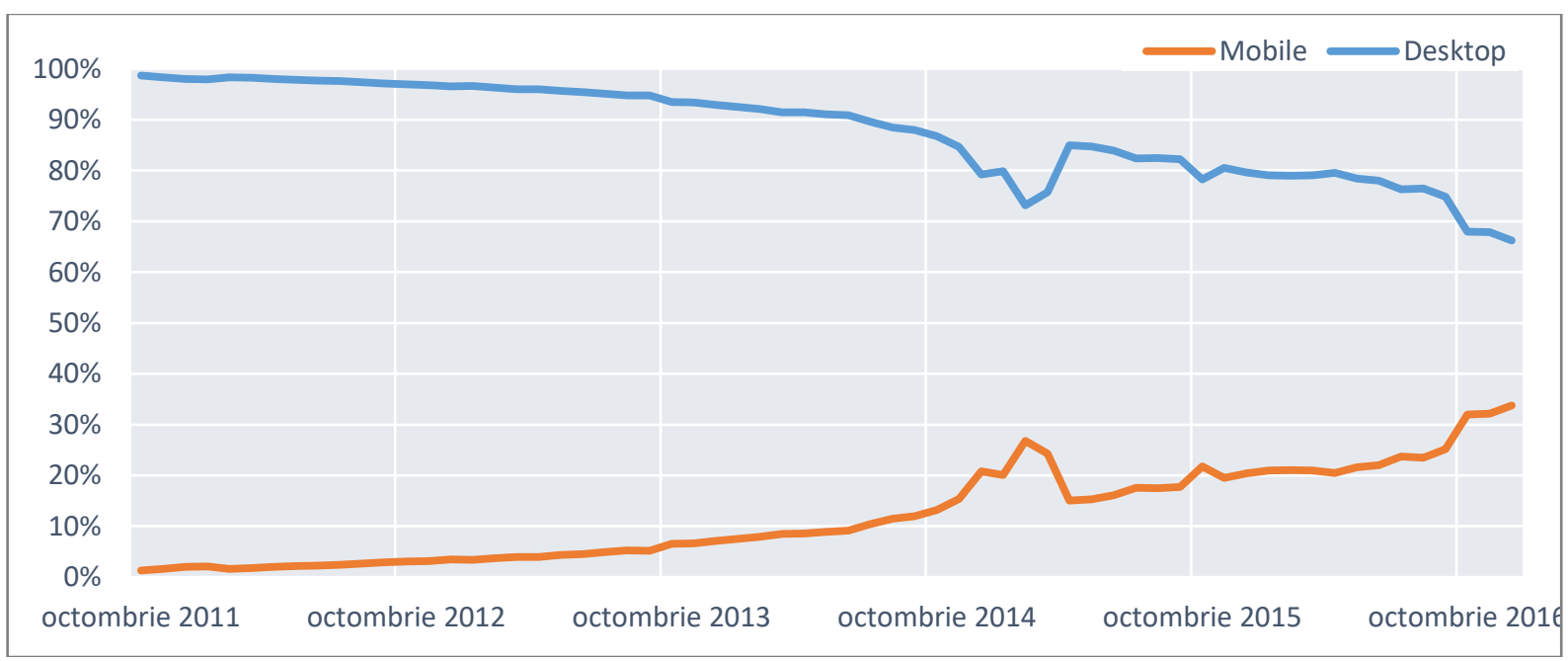

Fig. 6. Desktop vs. mobile Internet Usage in Romania. October 2011 - December 2016 trend. Data provided by statcounter.com

The most conservative European countries with respect to the use of mobile devices for accessing the Internet, i.e. countries where desktops are largely preferred, are: Ukraine (89.04\% desktop), the Czech Republic (88.97\% desktop), Russia (86.39\% desktop). There are, however, three countries where mobile Internet use surpassed desktop Internet use - which matches the global trend. It is the case of Turkey (61.24\% mobile), Poland $(58.46 \%)$ and Albania (52.53\% mobile).

7 Keeping Abreast of Time for FEAA Website Users

In order to have an overview of the use of mobile devices in the academic environment, we present a series of data obtained by monitoring traffic on the FEAA website over a fiveyear span, from October 2011 to December 2016 inclusive. The monitoring was done with the help of Google Analytics (analytics.google.com), and the data were then exported to and processed in Excel.

For a start, we will focus on data from the 2016, as they are current. Out of 553136 total sessions in 2016, 293046 came from desktop/laptop, 249998 from mobile phones and 10092 from tablets. In percentage, this is $52.98 \%$ desktop/laptop, $45.20 \%$ mobile phones, respectively $1.82 \%$ tablets (Figure 7). 


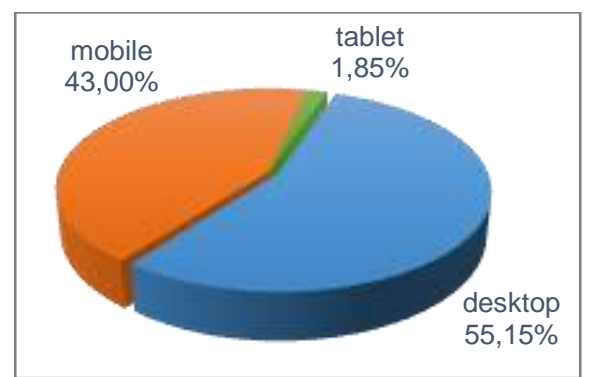

Fig. 7. Desktop, mobile and tablet access of feaa.uvt.ro in 2016

Analysing of the graph from 2016 reveals an interesting fact, that in September and October, the number of Internet access instances originating from mobile devices surpassed the number of Internet access instances on desktop/laptop for the first time. Next two month, in November and December, the positions were reversed (Figure 8).

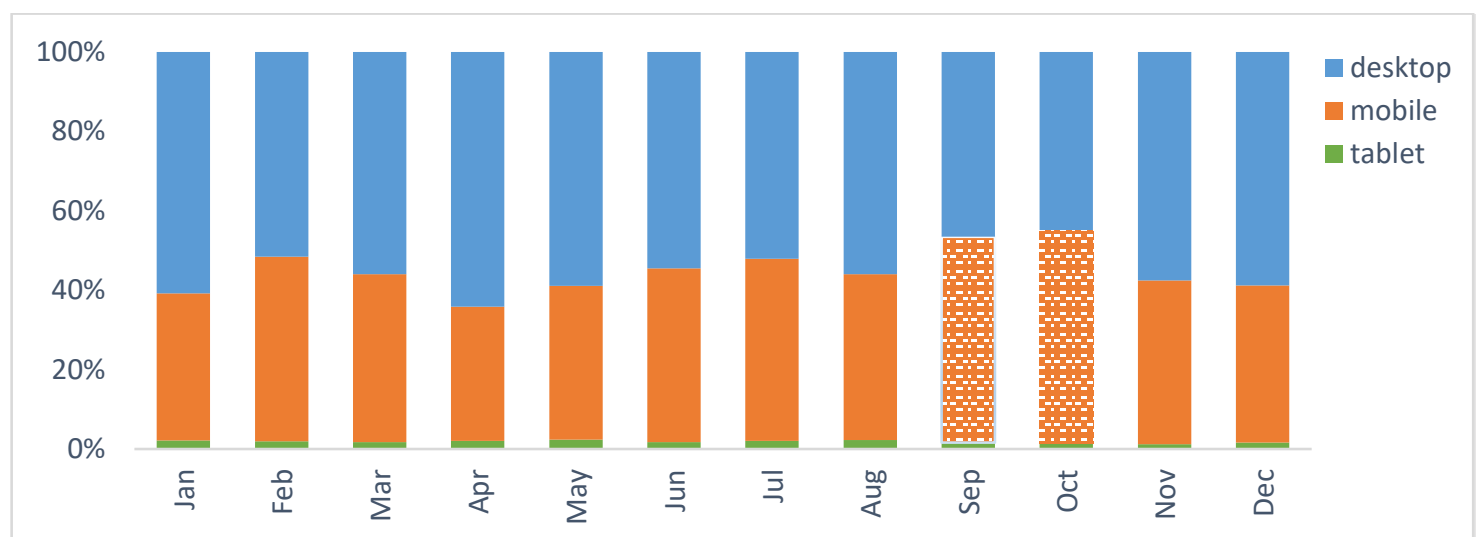

Fig. 8. Monthly distribution for desktop, mobile and tablet access of feaa.uvt.ro in 2016

These results might appear slightly surprising, but, when analyzing data from previous years, we can notice a similar trend in September and October. These two months are marked by an

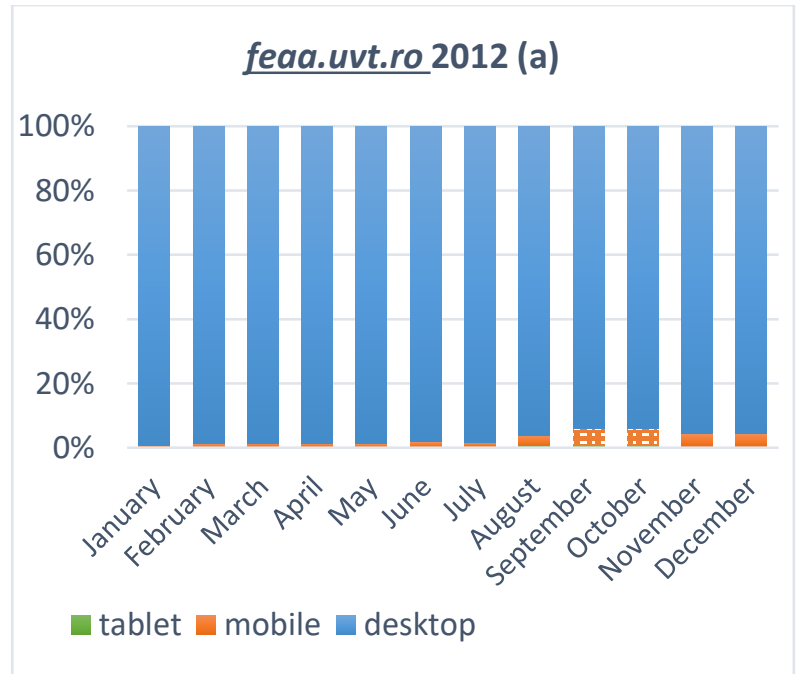

increase in mobile Internet access to a level that is superior to that recorded during the rest of the year (see Figure 9).

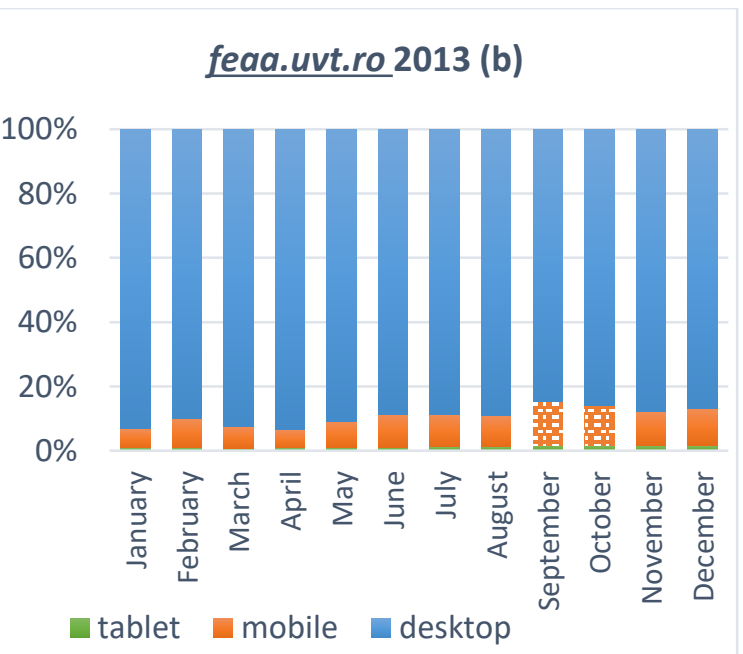




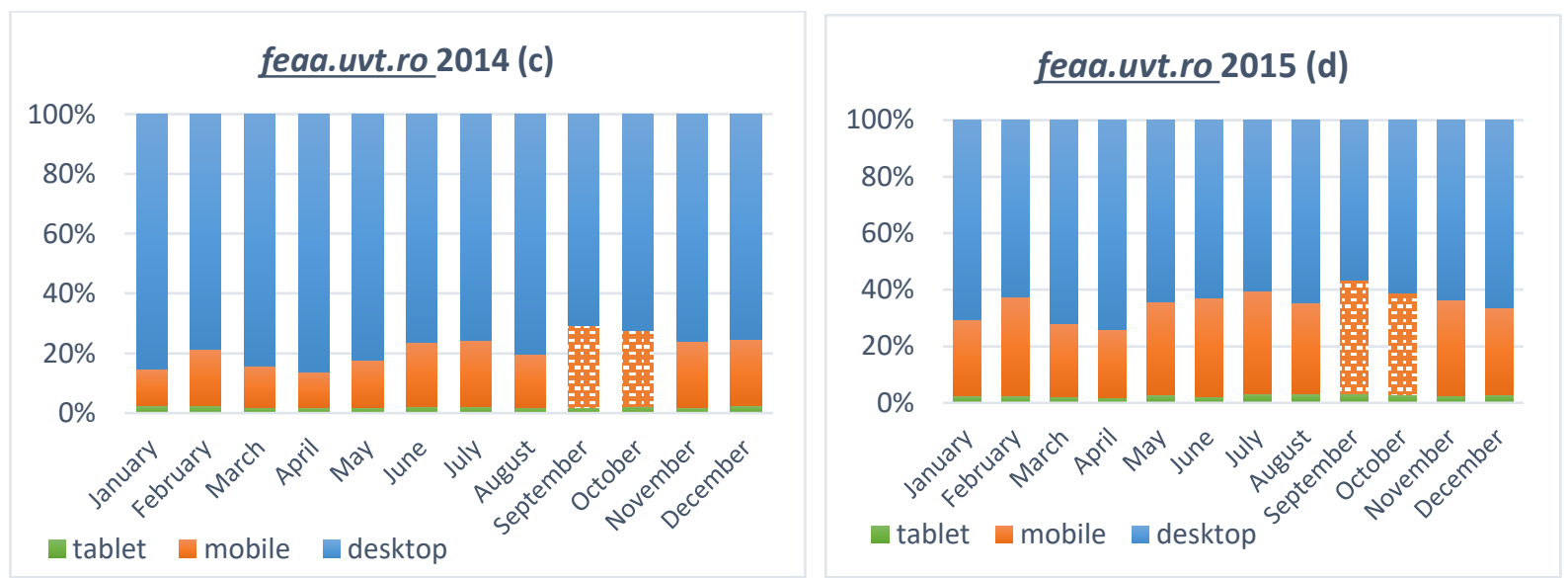

Fig. 9. Desktop and mobile access of feaa.uvt.ro. 2012 to 2015 yearly distribution

We may assume that this behavior is due to the fact that in September and October students travel more and keep checking the website for information regarding exams, registration, schedule, etc. Not being fully installed in the dorms or in rented apartments, they may not bring their computers (desktops/laptops) at this point, which accounts for the increased use of mobile devices to access studies-related information. Moreover, some students have no access or have limited access to the faculty's computers.

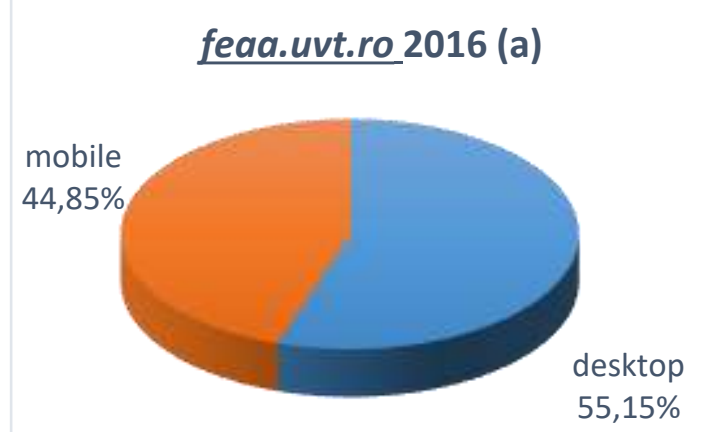

Upon comparing FEAA website access to the patterns at national level in 2016, one can notice that the percentage of mobile access of the FEAA website $(47,02 \%)$ is almost double the percentage of Internet access instances originating from mobile devices in Romania $(24,32 \%)$. The last three months of 2016 registered an increase not only in mobile access of the FEAA website $(49,86 \%)$, but also of websites in general at a national level $(32,61 \%)$.

\section{feaa.uvt.ro Oct-Dec 2016 (b)}

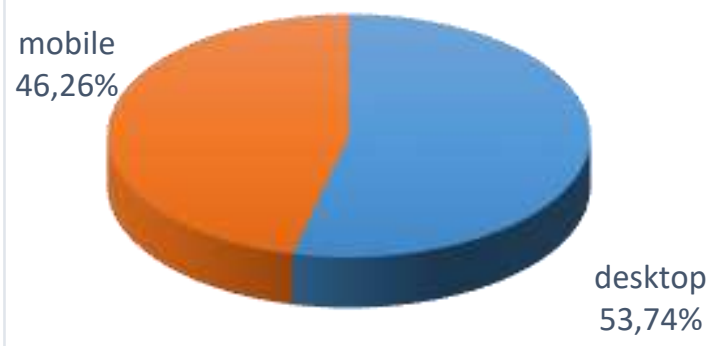

Fig. 10. Desktop vs mobile access of feaa.uvt.ro in 2016 (a) and in Oct-Dec 2016 (b)

On analyzing the data collected while monitoring FEAA website access from mobile devices in 2016, we could notice that Android had the lead $(68,12 \%)$, followed by iOS $(29,28 \%)$.

In order to give a general overview of the situation, the Figure 11 shows the percentages of desktop and mobile access from October 2011 to December 2016. One can clearly notice the downward trend in PC use as opposed to the upward trend in the use of mobile devices. Overall, the graph is similar in terms of trends and values to the worldwide StatCounter's graph presented in Figure. 


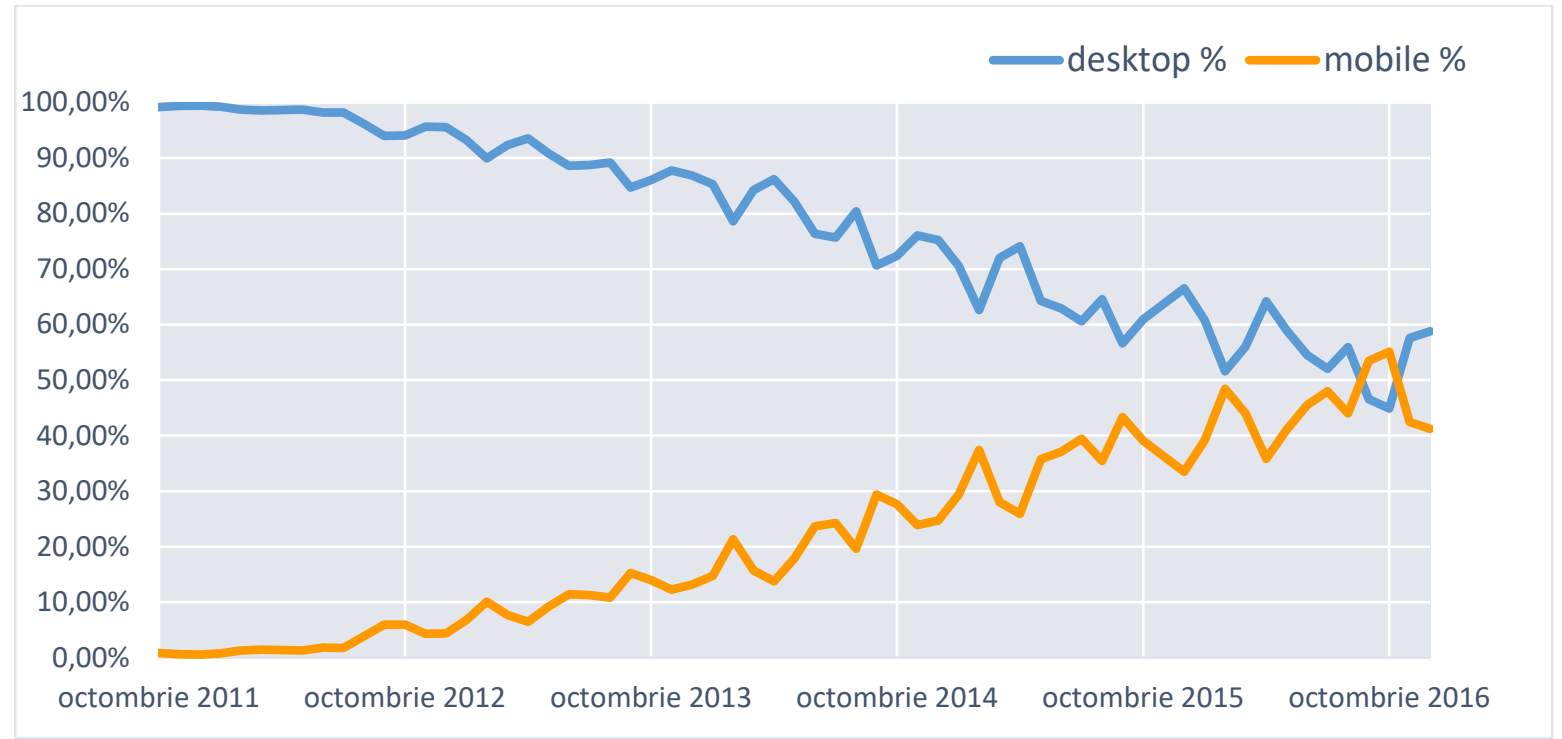

Fig. 11. Desktop and mobile access of feaa.uvt.ro.

October 2011 to December 2016. Monthly distribution

To see clearly the evolution of usage of mobile devices in these five years, we represented in the Figure 12 the average percentage of annual visits to the site FEAA, from 2011 to 2016: it started with a rate of $0.7 \%$ in mobile accesses in 2011 and continuously increased to $2.97 \%$ in $2012,10.94 \%$ in 2013 , $22.55 \%$ in $2014,36.86 \%$ in 2015 and $47.02 \%$ in 2016.

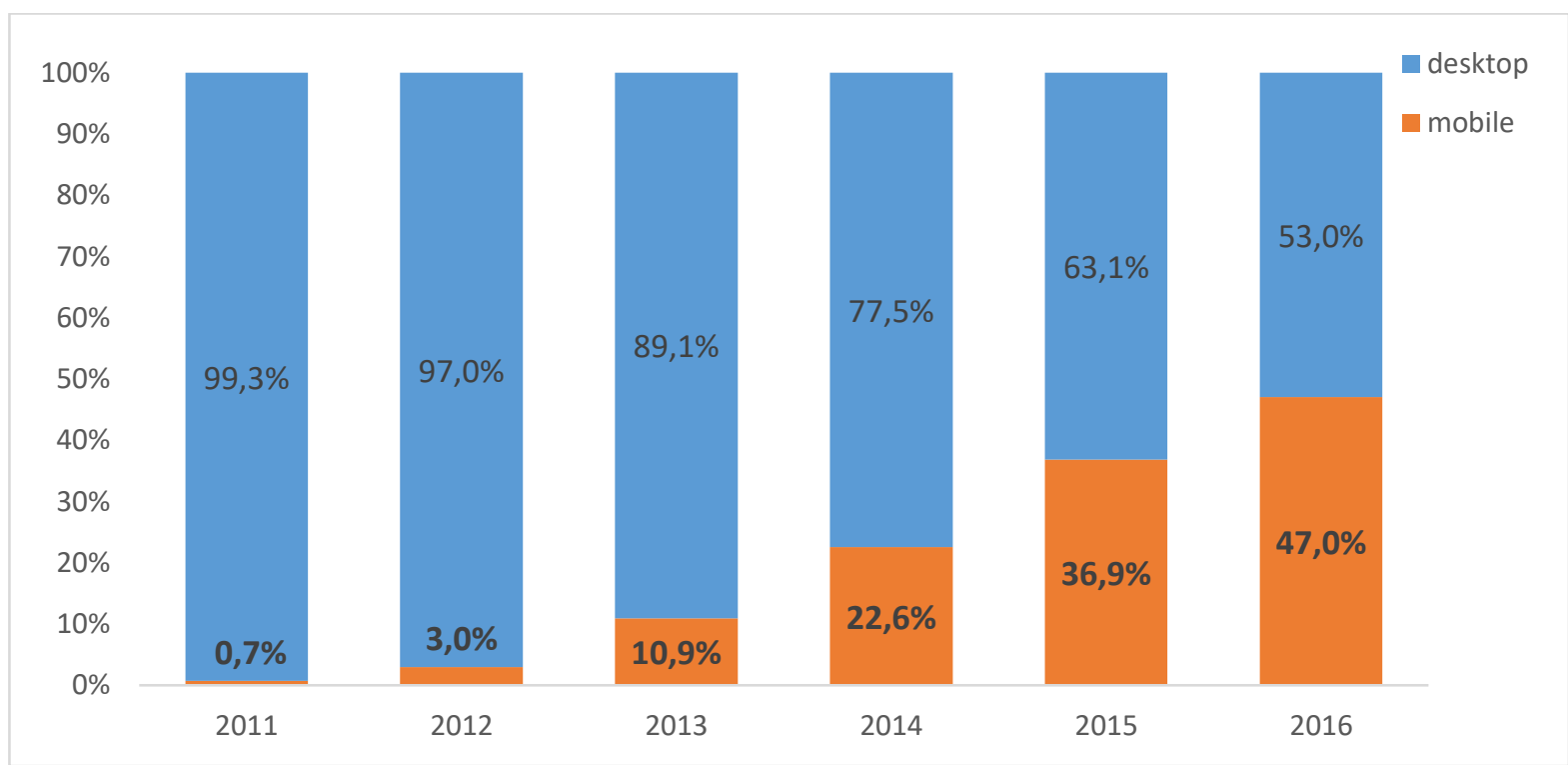

Fig. 12. Desktop and mobile access of feaa.uvt.ro. 2011 to 2016 yearly distribution

8 Mobile Internet Access - Where to? Conclusions

Looking back 10 years, when moment 0 happened (the launch of the iPhone in 2007), we believe that few of us could have imagined the magnitude of the mobile device trend.

The multiplication of high-speed mobile net- works, the introduction of increasingly powerful mobile devices on emergent markets explain how, for some people, smartphones have become more important than computers [21]. In fact, we are witnessing the birth of a new mobile era in which, as Google points out, "the world is full of mobile micro-moments" 
(www.thinkwithgoogle.com/collections/micromoments.html) - key moments when users interact with their mobile devices to do a search, to have fun, to communicate or to purchase something. Since every user is unique, the order and the occurrence of these activities vary, and the user's once linear online journey is obsolete [6].

As we have seen, Romania follows the global trends regarding the use of mobile devices to surf the Internet, and the youth is a step forward, as proven by the data on the FEAA website. The future will be all about mobile devices and, in our opinion, there is no way of reversing this trend.

Therefore, the main current priority for the online environment and especially for the business sector, is to have a mobile Web presence. Delivery and optimization of Web content on mobile devices will be the best way to reach the most profitable target markets. If several years ago, the saying was "If you are not online, you don't exist", nowadays it has become "If you are not mobile, you don't exist".

It is highly necessary to improve and optimize websites for mobile users, because they can no longer be ignored. At the same time, given that mobile devices account for more than half of Internet traffic, not only will these changes help companies successfully tackle changes, but they will also help them capitalize on the growing mobile market [1].

However, the race for mobile-friendly and mobile-first must not overlook website performance. A shift in priorities should leave aside objectives like website ranking and spectacular graphics and increase focus on content optimization and page speed and load time improvement on mobile devices. Because, as a rule, websites load slower on mobile devices than on desktop/laptop, ensuring a reasonable page speed for mobile websites will be paramount in improving user experience. Page speed will be the decisive factor in choosing the site by customers [5].

Mobile users must not be "punished" with heavy-content websites and features that have no purpose on small screens just to maintain both mobile and desktop under the same umbrella-RWD. Specific targeting by providing dynamic content depending on the users' devices seems to be more appropriate and able to create the best user experience and to increase conversion rate [12].

Mobile Web has much room for development when it comes to strategies, conversions or user experience. Measuring mobile moments that matter - by understanding and optimizing mobile traffic, mobile engagement, mobile conversion and mobile revenue - is essential for developing a successful mobile-first strategy [6].

\section{References}

[1] A. Betts, "Mobile Optimization and the Google Algorithm Change - 7 Steps to Stay Friendly," 01 April 2015. [Online]. Available: https://searchenginewatch.com/sew/how-to/2402354/mobileoptimization-and-the-google-algorithmchange-7-steps-to-stay-friendly. [Accessed December 2016].

[2] A. DiSilvestro, "Google's mobile-first index: how to prepare your business," November 2016. [Online]. Available: https://searchenginewatch.com/2016/11/14/googles-mobilefirst-index-how-to-prepare-your-business/. [Accessed December 2016].

[3] G. Sterling, "It's Official: Google Says More Searches Now On Mobile Than On Desktop," May 2015. [Online]. Available: http://searchengineland.com/its-officialgoogle-says-more-searches-now-on-mobile-than-on-desktop-220369. [Accessed January 2017].

[4] Official Google Blog, "Building for the next moment," May 2015. [Online]. Available: https://adwords.googleblog.com/2015/05/building-for-next-moment.html. [Accessed January 2017].

[5] J. Caiozzo, "Google's Mobile First Index: What It Means for the Future of SEO," December 2016. [Online]. Available: http://www.advancedwebranking.com/blog/google-mobile-indexwhat-it-means-for-seo/. [Accessed December 2016]. 
[6] J. Yu, "Google's shift to mobile-first: mobile moments that matter," November 2016. [Online]. Available: http://searchengineland.com/googles-shift-mobile-firstmobile-moments-matter-263971. [Accessed December 2016].

[7] Official Google Blog, "Rolling out the mobile-friendly update," April 2015. [Online]. Available: https://webmasters.googleblog.com/2015/04/rolling-outmobile-friendly-update.html. [Accessed January 2017].

[8] J. A. Saeteraas, "Are You Ready For Google's Mobile-Friendliness Rankings? Lessons From The Top 10,000 Sites," April 2015. [Online]. Available: https://www.scientiamo-

bile.com/page/are-you-ready-for-googlesmobile-friendliness-rankings-lessonsfrom-the-top-10000-sites. [Accessed December 2016].

[9] M. Jacobson, "What Does Mobile Friendly Mean? 6 Definitions," April 2015. [Online]. Available: http://www.dexmedia.com/blog/whatdoes-mobile-friendly-mean-6-definitions/. [Accessed January 2017].

[10] Official Google Blog, "Finding more mobile-friendly search results," February 2015. [Online]. Available: https://webmasters.googleblog.com/2015/02/findingmore-mobile-friendly-search.html. [Accessed January 2017].

[11] B. Schwartz, "Google Says There Are 4.7\% More Mobile-Friendly Websites Today Than Two Months Ago," April 2015. [Online]. Available: http://searchengineland.com/google-says-there-are-4-7more-mobile-friendly-web-sites-todaythan-two-months-ago-219487. [Accessed January 2017].

[12] C. Borodescu, "Google's Mobilegeddon Aftermath: Eight Months Into A Better Mobile Web," March 2016. [Online]. Available: https://www.smashingmagazine.com/2016/03/googles-mobilegeddon-aftermath-eight-months-better-mobile-web/. [Accessed December 2016].

[13] B. Meunier, "Mobilegeddon Is Beginning, Not Ending," May 2015. [Online].
Available: http://searchengineland.com/mobilegeddon-beginning-notending-220512. [Accessed January 2015].

[14] B. Schwartz, "Google: The Mobile Friendly Update Ranking Factor Will Be Real Time \& On A Page-By-Page Basis," March 2015. [Online]. Available: http://searchengineland.com/google-mobile-friendly-ranking-factor-runs-realtime-page-page-basis-216100. [Accessed January 2017].

[15] Official Google Blog, "Mobile-first Indexing," November 2016. [Online]. Available: https://webmasters.googleblog.com/2016/11/mobile-first-indexing.html. [Accessed January 2017].

[16] Google Developers, "Mobile SEO Overview," May 2016. [Online]. Available: https://developers.google.com/webmasters/mobile-sites/mobile-seo/. [Accessed January 2017].

[17] Anne-Marie, "Google's New Definition of 'Mobile-Friendly'," May 2015. [Online]. Available: https://www.designprintdigital.com/digital-media/googles-newdefinition-of-mobile-friendly/. [Accessed January 2017].

[18] YOOtheme GmbH, "Warp Framework," october 2016a. [Online]. Available: http://yootheme.com/themes/warp-framework. [Accessed october 2016].

[19] C. Borodescu, "State of the Mobile Web - 4 Out of 10 Sites Are Oblivious To Google's New Mobile Ranking Signal," June 2015. [Online]. Available: https://www.appticles.com/blog/2015/06/ state-of-the-mobile-web-4-out-of-10sites-are-oblivious-to-googles-new-mobile-ranking-signal/. [Accessed December 2016].

[20] R. Simpson, "Mobile and tablet internet usage exceeds desktop for first time worldwide," November 2016. [Online]. Available: http://gs.statcounter.com/press/mobile-and-tablet-internetusage-exceeds-desktop-for-first-timeworldwide. [Accessed December 2016].

[21] J. Titcomb, "Mobile web usage overtakes desktop for first time," November 2016. 
[Online]. Available: http://www.telegraph.co.uk/technology/2016/11/01/mobile-web-usage-overtakes-desktop-forfirst-time/. [Accessed December 2016].

[22] Philipp, "Why 'mobile first' is the new 'responsive'," 19 August 2013. [Online]. Available: http://metamonks.com/mobilefirst-vs-responsive/. [Accessed December 2016].

[23] R. Graham, "Mobile First: What Does It Mean?," 5 March 2012. [Online]. Available: http://www.uxmatters.com/mt/archives/2012/03/mobile-first-what-does-itmean.php. [Accessed December 2016].

[24] CMVBlog, "Mobile First Design: Why It's Great and Why It Sucks," December
2016. [Online]. Available: https://codemyviews.com/blog/mobilefirst.

[25] N. Sherlock, "Why Google, Facebook Thrive on a Mobile First Strategy, And You Will Too," August 2016. [Online]. Available: https://www.inspireteam.com/2016/08/23/why-google-facebook-thrive-on-a-mobile-first-strategyand-you-will-too/. [Accessed December 2016].

[26] T. Jensen, "How To Use Analytics To Build A Smarter Mobile Website," March 2014. [Online]. Available: https://www.smashingmagazine.com/2014/03/how-to-use-analyticsto-build-a-smarter-mobile-website/. [Accessed December 2016].

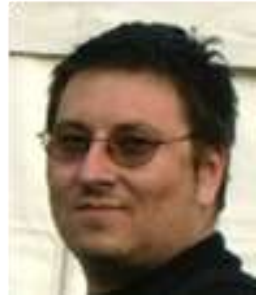

Romeo MARGEA has graduated the Faculty of Physics, and the Faculty of Economics and Business Administration, from the West University of Timisoara. From 2008 he holds a PhD diploma in Economics. He is currently lecturer within the Business Information Systems Department, Faculty of Economics and Business Administration from the West University of Timisoara. $\mathrm{He}$ is interested in web design, web development, CMS, SEO, open source software.

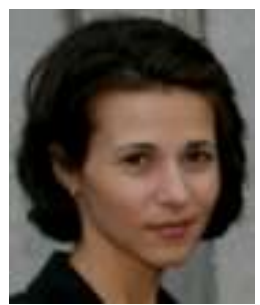

Camelia MARGEA has graduated the Faculty of Economics, at West University of Timisoara. Since 1997 then she joined to the teaching staff of the Faculty of Economics and Business Administration, within the West University of Timisoara, in the Business Information Systems Department. Her scientific fields of interest include: eLearning, multimedia design, open source software, human resource management. 